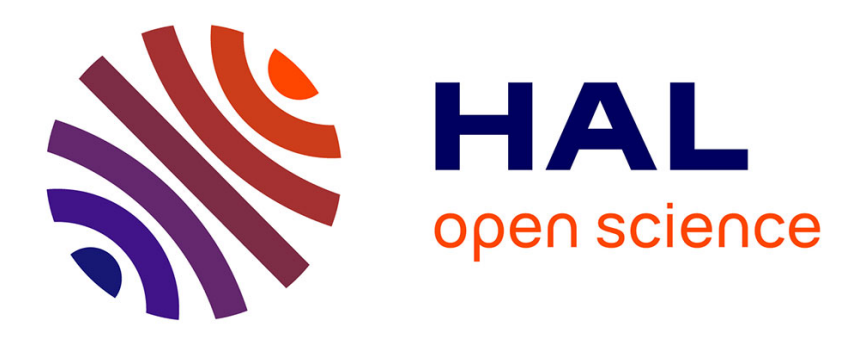

\title{
Vater- und Mentorfiguren bei Robert Walser. Dialog, Mimikry und Subversion
}

\author{
Konrad Harrer
}

\section{To cite this version:}

Konrad Harrer. Vater- und Mentorfiguren bei Robert Walser. Dialog, Mimikry und Subversion. Etudes Germaniques, 2017, Robert Walser. Dialogues, 285 (1), pp.21-38. 10.3917/eger.285.0021 . hal-02559728

\author{
HAL Id: hal-02559728 \\ https://hal.univ-lorraine.fr/hal-02559728
}

Submitted on 5 Oct 2020

HAL is a multi-disciplinary open access archive for the deposit and dissemination of scientific research documents, whether they are published or not. The documents may come from teaching and research institutions in France or abroad, or from public or private research centers.
L'archive ouverte pluridisciplinaire HAL, est destinée au dépôt et à la diffusion de documents scientifiques de niveau recherche, publiés ou non, émanant des établissements d'enseignement et de recherche français ou étrangers, des laboratoires publics ou privés. 


\section{Vater- und Mentorfiguren bei Robert Walser: Dialog, Mimikry und Subversion}

Wer vom Dialogischen in Robert Walsers Werk sprechen will, widmet sich einem heiklen Thema. Ist es nicht eher der Monolog, der für Walsers Schreiben - und für das Reden der Walserschen Protagonisten - charakteristisch ist? Der wuchtige Wortschwall des jungen Simon Tanner, der sich von den anderen nichts sagen lässt und viel eher Lektionen erteilt als entgegennimmt, frappiert den Leser etwa zu Beginn der Geschwister Tanner. Und dieser Wortschwall richtet sich eben an ältere Männer, die es Simon Tanner erlauben könnten, den Fuß ins Berufsleben zu setzen. Er richtet sich insofern an potentielle Vaterfiguren, wenn man davon ausgeht, dass der Vater herkömmlicherweise als derjenige verstanden wird, der den Sohn ins Leben führt und ihm als Vorbild dient. Damit stellt sich die Frage nach den Modalitäten einer «Nachfolge», einer Mimesis väterlichen Handelns und/oder einer eventuellen Distanznahme. An Simon Tanner ist zu sehen, dass es mit einer solchen Nachfolge nicht weit her ist - ja, dass es letztlich nicht einmal zu einem Dialog kommt, der ein entsprechendes Verhalten einleiten könnte. In seinem Aufsatz «Wie weise ist Robert Walsers Weisheit» thematisiert Peter von Matt die Beziehung der Walserschen Protagonisten zu ihren «Mentoren », wie er sie nennt: «Was aber tut der Walserjüngling? Er nimmt dem Mentor auf der Stelle das Wort aus dem Mund und hält die Mentorrede selbst, als Gegenrede, rhetorisch so hochgemut wie das Vorbild und gleichermaßen gespickt mit Weisheiten und Lebenslehren. » ${ }^{1}$ Das Vorbildhafte der Vaterfiguren wird konterkariert, in Geschwister Tanner durch (wohlgemerkt: rhetorische) Imitation, ja sogar Überbieten: man kann es selbst und weiß es besser. Dahinter steckt eine tiefe Verunsicherung und ein Aufbäumen gegen diese Verunsicherung; das erfahren wir in Jakob von Gunten, wo sich die Selbstsicherheit in ihr Gegenbild verkehrt (oder zu verkehren scheint): Jakob hat sein Vaterhaus verlassen, weil er «gefürchtet hätte, von seiner [das heißt seines Vaters, K.H.] Vortrefflichkeit erstickt zu werden $»^{2}$.

Während traditionelle Vater- und Mentorenfiguren zurückgewiesen werden, weckt eine andere Person Jakobs Interesse: Benjamenta, der Direktor einer Dienerschule, in die er eintritt. In ihm findet Jakob einen unbürgerlichen Anti-Mentor, der Jakob das Klein- bzw. Nichts-Sein nahebringt - als Abwehrreaktion gegen die Anforderungen der bürgerlichen Gesellschaft. Indem Benjamenta als unbürgerliche Vaterfigur gezeichnet ist, entspricht er in seiner Natur dem Bild des Vaters, wie Walser es in tendenziell autobiographischen Texten vermittelt - Texten, denen sich Peter von Matt in einem anderen Artikel widmet, nämlich in

\footnotetext{
${ }^{1}$ Peter von Matt : «Wie weise ist Robert Walsers Weisheit?», in : Id. : Das Kalb vor der Gotthardpost, München : Hanser, 2012, S. 226. (Erstabdruck in W. Groddeck, R. Sorg, P. Utz und K. Wagner (Hrsg.) : Robert Walsers «ferne Nähe ». Neue Beiträge zur Forschung, München : Fink, 2007.)

${ }^{2}$ Robert Walser : Jakob von Gunten, Frankfurt a. M. : Suhrkamp, 1985 (in der Folge im Haupttext sigliert als JvG mit Seitenangabe), S. 12.

* Konrad HARRER, Maître de Conférences, Etudes Germaniques, Université de Lorraine (site de Nancy), 13, rue de Lailletas, F-40990 Saint-Paul-lès-Dax ; courriel : konrad.harrer@univ-lorraine.fr
} 
«Die Schwäche des Vaters und das Vergnügen des Sohnes » ${ }^{3}$. Mit Bezug auf Prosastücke wie Bild des Vaters zeichnet von Matt das Porträt eines Mannes, der sich als Handelstreibender versucht (ursprünglich war er Handwerker, nämlich Buchbinder), aber aufgrund fehlenden kommerziellen Geschicks, vor allem fehlender Dynamik und Tüchtigkeit, ins wirtschaftliche Abseits gerät. Von Matt interpretiert diese Haltung auf zweierlei Art: Zum einen sieht er in dieser Person eine vorkapitalistische Gemütlichkeit überleben, die noch in Gottfried Kellers Seldwyla porträtiert werde. Zum anderen interpretiert er den Niedergang psychoanalytisch: als Realisierung der vom ödipal motivierten Sohn gehegten Wünsche: «Vor den Augen des Knaben ging der böse Wunsch in Erfüllung. Die Schwäche des Vaters war, so musste es scheinen, die Tat des Sohnes [...] ». (Ibid. S. 193) Die Schuld werde später abgegolten durch Walsers eigenen sozialen Niedergang. Aber wie bei dem Vater sei der soziale Niedergang kein existenzieller: weil das Selbstwertgefühl sich bei den beiden eben nicht von der gesellschaftlichen Stellung ableite. Insofern ließe sich also auch hier von einer mimetischen Beziehung des Sohnes zu seinem Vater sprechen. Er ahmt den Vater nach - aber eben nicht im bürgerlichen Sinn: Es ist eine Nachfolge nicht in das Leben, das bürgerliche Leben, sondern aus diesem Leben heraus. Doch - wohlgemerkt - wird dieser Schritt nicht gemeinsam getan. Die Annäherung von Vater und Sohn erfolgt a posteriori; von einem Dialog, einem Austausch zwischen den beiden kann nicht die Rede sein (weder in Walsers Biografie noch in den explizit autobiographischen Texten).

Wenn irgendwo von einem echten Dialog gesprochen werden kann, dann noch am ehesten in der reinen Fiktion. Wie sieht es in den beiden Romanen aus, in denen Vaterfiguren eine zentrale Rolle spielen?

\section{Der Gehülfe}

Während sich im Fall von Jakob von Gunten bei dieser Fragestellung, wie wir sehen werden, die Geister scheiden, herrscht in der Interpretation des Gehülfen eine gewisse Einhelligkeit: Die Persönlichkeiten des Ingenieurs Tobler und Joseph Martis, der nicht nur beruflich als dessen Untergebener fungiert, sondern sich - entsprechend dem Sozialmodell des «ganzen Hauses ${ }^{4}$ - auch in die Familie, dessen selbstherrliches Oberhaupt Tobler ist, integriert sieht, scheinen so unterschiedlich zu sein, dass eine Annäherung zwischen den beiden unmöglich anmutet. Und doch haben sie bei genauerem Hinsehen vieles gemein - eben das Unbürgerliche, das Träumerische, das sich auch im projekteschmiedenden Tobler entfaltet, oder das Genießerische - Tabak und Alkohol seien repräsentativ genannt - das seitens Toblers nicht als Lebensgenuss reicher, aber kalt kalkulierender Kapitalisten verstanden werden darf, sondern als ordnungszerstörende Impulsivität und als Fluchtreaktion vor den Anforderungen des Lebens (vgl. Toblers Wirtshausbesuche, die die notwenigen geschäftlichen Aktivitäten ersetzen). Joseph ahnt diese Gemeinsamkeiten und hängt an Tobler

\footnotetext{
${ }^{3}$ Peter von Matt : «Die Schwäche des Vaters und das Vergnügen des Sohnes. Über die Voraussetzungen der Fröhlichkeit bei Robert Walser », in : Klaus-Michael Hinz und Thomas Horst : Robert Walser, Frankfurt a. M. : Suhrkamp, 1991, S. 182-198. (Erstabdruck in Neue Rundschau 90, 1979, S. 197-213.)

${ }^{4}$ Diesem Aspekt widmet Karl Wagner (Herr und Knecht. Robert Walsers Roman Der Gehülfe, Wien : Braumüller, 1980) ein ausführliche Darstellung (S. 99-128).
} 
trotz dessen abweisendem Wesen. ${ }^{5}$ Die Aussicht auf eine Beziehung des menschlichen Austauschs und der Solidarität wird immer plausibler, je mehr sich der Untergang des Hauses Tobler konkretisiert, und der Roman suggeriert Josephs Hoffnung, dass der Ruin seine Sehnsucht nach Integration (in die Familie Tobler) und Freundschaft (mit Tobler) in Wirklichkeit verwandelt. ${ }^{6}$ In einer Gesellschaft, in der Tobler letztendlich ebenso desorientiert ist und scheitert wie Joseph, ${ }^{7}$ wird die Solidarität unter den Scheiternden, unter den Kleinen (wie groß sie zunächst auch scheinen wollen) als Möglichkeit denkbar: Tobler erweist sich als ebenso ohnmächtig wie sein «Gehülfe », und verliert damit den Status, der eine Beziehung zwischen Gleichgestellten verhinderte. Das ahnt Joseph und deshalb scheint ihm hier die Überwindung jener Ängste denkbar, die alle Walserschen Protagonisten - mehr oder weniger deutlich - heimsuchen: das Erdrücktsein durch die so vorbildlichen Väter. Ja, Joseph wähnt sogar eine Art Seelenverwandtschaft bzw. eine ähnliche Art der Lebensführung zwischen ihm und Tobler zu erkennen: «Ich habe mich in die hiesigen, eigenartigen Verhältnisse hineingefunden, und ich glaube, ich passe in diese Verhältnisse. ${ }^{8}$ Joseph meint eine Welt gefunden zu haben, die seiner Natur entspricht und es dieser erlaubt, sich auszuleben, weil sie in Einklang mit den Menschen ist, die ihn umgeben: «Wie ist es mir möglich, zu leben, ohne Dummheiten zu begehen? [...] Hier durfte ich 'kopflos' sein, wenigstens bis zu einem gewissen Grade. » (DG 239 und 240) Unfähig, den Forderungen der bürgerlich-neuzeitlichen Rationalität zu entsprechen, hat Joseph in der Villa Abendstern eine Welt gefunden, wo diese Forderungen nicht ihre unumschränkte Herrschaft angetreten haben. Wie sehr Joseph an der Villa Abendstern und ihren Bewohnern hängt, zeigt der Wille zu bleiben, der gegen Ende des Romans eher noch größer wird, wo die Umstände ihn doch eher dazu anhalten müssten, sich eine andere Stellung zu suchen. Er sieht in seinem Posten bei Tobler eine «Lebensstellung » (DG 192) und weist den Gedanken eines Weggangs lange von sich. ${ }^{9}$ Als diese Möglichkeit dann tatsächlich Wirklichkeit zu werden droht, ja unmittelbar bevorsteht, weigert er sich, das Unabwendbare ins Auge zu fassen, so als ob diese Vogel-Strauß-Haltung es ihm erlauben

\footnotetext{
${ }^{5}$ Darauf weisen u.a. Dieter Borchmeyer (Dienst und Herrschaft. Ein Versuch über Robert Walser, Tübingen : Niemeyer, 1980, S. 47) und Dagmar Grenz (Die Romane Robert Walsers. Weltbezug und Wirklichkeitsdarstellung, München : Fink, 1974, S. 221) hin.

${ }^{6}$ Lukas Rüsch (Ironie und Herrschaft. Untersuchungen zum Verhältnis von Herr und Knecht in Robert Walsers Roman Der Gehülfe, Königstein im Taunus : Forum Academicum in der Verlagsgruppe Athenäum, Hain : Hanstein, 1983, S. 128) sieht hier die Hoffnung Jakobs, « es könnten in der Ausnahmesituation des Bankrotts Konventionen fallen - so daß sich am Ende seine Abhängigkeit in ein persönliches Vertrauensverhältnis verwandelt. »

${ }^{7}$ In seiner Rezension von Siegfried Kracauers Angestellten weist Walter Benjamin auf die (potentielle) Nähe von Unternehmern und Angestellten in der von ihm beschriebenen Form des Kapitalismus hin: « So eingreifend seine Kritik am Unternehmertum ist, es teilt für ihn, als Klasse betrachtet, mit der ihm untergebenen den Charakter des Subalternen zu sehr, um als eigentlich bewegende Kraft und zurechnungsfähiger Kopf im Wirtschaftschaos anerkannt zu werden. » (Walter Benjamin: «Politisierung der Intelligenz », in: Siegfried Kracauer : Die Angestellten, Frankfurt a. M. : Suhrkamp, 1971, S. 121). In Walsers Roman wird allerdings kaum die Fragilität des Unternehmertums als solches dargestellt, sondern die Außenseiterposition Toblers in diesem Milieu: Gezeigt wird der Unterschied zwischen gewieften Financiers (wie dem « Kapitalisten » namens Fischer, der eines Tages in Toblers Domizil auftaucht) und ohnmächtigen Möchtegern-Unternehmern wie Tobler, die die Spielregeln des Kapitalismus nicht beherrschen.

${ }^{8}$ Robert Walser : Der Gehülfe, Frankfurt a. M. : Suhrkamp, 1985 (in der Folge im Haupttext sigliert als DG mit Seitenangabe), S. 185.

${ }^{9}$ Vgl. DG 280, wo Joseph Frau Tobler ins Wort fällt: «'Lassen Sie mich die Gelegenheit ergreifen, Ihnen zu raten, wenn Sie einmal von uns fort sind - ' Aber ich gehe ja gar nicht fort!' - »
} 
würde, bis zum letzten Ende die Freuden auszukosten, ein Mitglied des Hauses Tobler zu sein: « [...] 'wozu es herbeiziehen', dachte er, 'es kommt schon von selber'. » (DG 283)

Doch alle diese Bemühungen verhindern nicht, dass der Wunsch, freundschaftliche Beziehungen zu Tobler herzustellen, zum Scheitern verurteilt ist. Kein Abschnitt im Text bringt dies deutlicher und gleichzeitig schlichter zum Ausdruck als die Beschreibung des Heiligen Abends, den Toblers und Joseph zusammen verbringen: «Ja, wie einsam waren diese drei Menschen [...]. »(DG 275) Einsam, isoliert sind alle drei - in Bezug auf ihr soziales Umfeld, aber eben auch untereinander; Joseph am meisten: « [...] am einsamsten der Gehülfe [...]. » (Ibid.) Der Text fügt folgende Erläuterung bei: «[...] weil er nicht, wie Herr Tobler, sagen durfte, er habe das Recht, in diesem Hause zu tun und zu verhindern oder zu umgehen, was ihm beliebe [...]. » (Ibid.) Mit anderen Worten: aus dem Scheitern entsteht nicht notgedrungen eine Solidarität zwischen den Opfern, es schmiedet sie nicht zusammen gegen den gemeinsamen Gegner. Der Machtkampf geht weiter trotz des Niedergangs Toblers, der in keinem Moment auf seine Rolle als Herr und Meister zu verzichten gedenkt. Schon zu Beginn des Romans wohnt der Leser einer Auseinandersetzung ${ }^{10}$ bei, welche erahnen lässt, wie schwierig die Beziehungen zwischen den beiden Menschen sein werden. Joseph selbst ist darauf gefasst: «Möglich, daß es zwischen uns eines Tages persönliche Keilereien gibt. » (DG 19)

Dass es zu einem wahren Dialog nicht kommt, liegt unter anderem an der zweideutigen Haltung Josephs, der keineswegs der hilfreiche Gehülfe ist, der er sein sollte, sondern sich, sobald sein Herr abwesend ist, alle Freiheiten herausnimmt und seine Arbeit vernachlässigt. Hinzu kommt eine «ödipale Konstellation » ${ }^{11}$, in der sich Joseph nicht als potentieller Freund oder Bruder, sondern als Rivale Toblers im Kampf um die Zuneigung von dessen Frau erweist.

Das Haupthindernis für eine freundschaftliche Beziehung dürfte jedoch die Verständnislosigkeit Toblers sein, der als Person mit völlig fehlender Hellsicht bezüglich seiner materiellen Lage und seiner psychisch-geistigen Veranlagung erscheint. Mangelnde Selbsterkenntnis ist bei ihm noch viel mehr als bei Joseph $\mathrm{zu}$ diagnostizieren, vermag Letzterer doch wenigstens teilweise seine «Fehler» $\mathrm{zu}$ erkennen und genug Gemeinsamkeiten zwischen sich und seinem Chef wahrzunehmen, um ihn liebzugewinnen (denn die Anziehungskraft, die Frau Tobler auf ihn ausübt, erklärt nicht allein, warum Joseph bei der Familie bleiben will). Herr Tobler seinerseits verspürt nie das Bedürfnis (im Gegensatz zu Benjamenta), sich Joseph zu eröffnen, eine Beziehung des Vertrauens aufzubauen oder ganz einfach eine Seelen- oder Geistesverwandtschaft einzugestehen. Von Anfang bis Ende des Romans sieht er sich als Herr; Christoph Siegrist folgert somit zu Recht: « Das Herr-Knecht-Verhältnis entfaltet keinerlei Dynamik, beide verharren in ihren Rollen, es besteht keinerlei Aussicht auf Befreiung [...]. » ${ }^{12}$ Tatsächlich ist es so, dass Joseph Marti

\footnotetext{
${ }^{10} \mathrm{Vgl}$. den rüden Ton, in dem Joseph empfangen wird: "Warum', sagte er, Joseph strafend anblickend, 'kommen Sie denn eigentlich heute schon?' » (DG 8)

${ }^{11}$ Lukas Rüsch : Ironie und Herrschaft (Anm. 5), S. 144.

12 Christoph Siegrist : «Robert Walser: Der Gehülfe (1907)», in : Paul Michael Lützeler (Hrsg.) : Deutsche Romane des 20. Jahrhunderts, Königstein/Taunus : Athenäum, 1983, S. 57.
} 
« den Launen seines Herrn ausgeliefert ${ }^{13}$ bleibt, und in den Auseinandersetzungen « zieht er doch am Ende den kürzeren. ${ }^{14} \mathrm{Zu}$ bedenken ist jedoch: Es gibt eine Dynamik, aber sie liegt ausschließlich in Josephs Geist, der immer mehr an sein Herrschaftshaus gefesselt wird. Sie fehlt völlig in der Interaktion zwischen Herr (bzw. Herrin) und Diener, da erstere - Frau Tobler im Bereich der Erotik, Herr Tobler als potentieller Freund - nicht 'mitspielen'. Der Herr, der seinen Untergebenen oft 'anherrscht', ist unfähig, zu einer anderen Sprache, der Sprache der Intimität, des menschlichen Verstehens, zu finden. Enttäuscht von diesem Verhalten, aber auch die Entfremdung verspürend, der dieser zugleich ohnmächtige und selbstverliebte Mensch zum Opfer fällt, ist Joseph zwischen Mitleid und Verachtung hin- und hergerissen: Mitleid für einen Menschen, dem jede Luzidität abgeht und der aus diesem Grund in sein Verderben rennt, Verachtung für einen Mann, der seiner Familie, die er tyrannisiert, und Joseph selbst wehtut, für dessen Zuneigung er völlig blind ist. Dieser Gefühls-Mix, wie er sich in Joseph ausgeformt hat, zeitigt eine sehr zwiespältige Haltung als Antwort auf die herrscherliche, unfreundliche, ja 'unfreundschaftliche' Haltung Toblers - und macht es Joseph unmöglich, seinem Chef gegenüber eine reine, lautere Freundschaft zu empfinden - trotz der Liebe, die er spürt, die er aber in den «Grunde seines Herzens » verbannen muss :

Er bemitleidete Tobler, er verachtete ihn, und er fürchtete sich zugleich vor ihm. Das waren drei sehr häßliche Empfindungen, eine wie die andere natürlich, aber auch ungerecht. Was veranlaßte ihn, nun noch länger der Angestellte dieses Mannes zu bleiben? Der Gehalt-Rückstand? Ja, das auch. Aber es war noch etwas ganz anderes, etwas Wichtigeres: er liebte aus dem Grunde seines Herzens diesen Menschen. (DG 283)

\section{Jakob von Gunten}

Verglichen mit dem Gehülfen ist die Aussicht auf freundschaftliche Verhältnisse in Jakob von Gunten natürlich um einiges naheliegender - und tatsächlich glaubt Peter von Matt in diesem Roman eine „Urversöhnung“'15 zwischen der Vaterfigur Benjamenta und dem 'Sohn’ Jakob zu erkennen. Wie steht es damit? Entwickelt sich im Roman eine herzliche, freundschaftliche Beziehung zwischen Benjamenta und Jakob, so dass dieses Werk tatsächlich zum Fluchtpunkt des Vater-Sohn-Dialogs bei Robert Walser würde? Schon in den ersten Seiten erscheint der Gedanke, dass die Beziehung zwischen Benjamenta und Jakob auf Dauer angelegt ist, dass sich ein Band knüpft, welches nicht mehr reißen soll: «[...] ich fühlte mit einemmal, daß ich da nicht mehr loskäme. » (JvG 12) Indem er Benjamentas Welt und Benjamentas Wesen zu erforschen sucht, durchbricht Jakob die von dem scheuen, sich (wie er selbst!) in eine Unerreichbarkeit flüchtenden Mann errichteten Barrieren. Der Direktor eröffnet sich dem

\footnotetext{
${ }^{13}$ Ibid. S. 53: «Das Herrschaftsverhältnis läßt sich nicht abbauen, er bleibt als Abhängiger dem guten Willen, ja den Launen seines Herrn ausgeliefert, welche ihn dies zeitweilig vergessen lassen können, um es ihn alsbald umso schmerzlicher spüren zu lassen. »

${ }^{14}$ Dagmar Grenz: Die Romane Robert Walsers (Anm. 4), S. 180: «Joseph aber ist immer der Unterlegene; selbst wenn er es einmal wagt, mutig zu sein, zieht er doch am Ende den kürzeren. »

${ }^{15}$ Peter von Matt : «Die Schwäche des Vaters und das Vergnügen des Sohnes » (Anm. 3), S. 197.
} 
Eleven und gibt - im Glauben an eine Seelenverwandtschaft ${ }^{16}$ - einem Gefühl der Freundschaft, ja der Liebe in sich Raum: «Ich habe eine seltsame, eine ganz eigentümliche, jetzt nicht mehr zu beherrschende Vorliebe für dich gewonnen. »(JvG 94) Die an dieser Stelle noch etwas zurückhaltende Formulierung - Benjamenta wird später deutlichere Worte finden ${ }^{17}$ - ist dennoch in diesem Kontext explizit genug, damit wir in dieser Hinsicht eine klare Unterscheidung zwischen dem Gehülfen und Jakob von Gunten treffen können: Während in ersterem tatsächlich eine affektive Nähe des Protagonisten zu seinem Vorgesetzten (und seiner Frau) entsteht, ist es in letzterem der Vorgesetzte, der die stärkeren Gefühle zum Untergebenen empfindet - und vor allem: der sie mit der größeren Offenheit, Freimütigkeit äußert. Tatsächlich ist es so, dass Jakob, auch wenn er sich angezogen fühlt, seine Gefühle nie so deutlich zum Ausdruck bringt, weder in der Interaktion mit Benjamenta, noch in den Tagebucheinträgen, aus denen der Roman besteht. Benjamenta, im Gegensatz zu Tobler, ist am Ende des Romans der intimen Diskursform fähig. Er verspürt das Bedürfnis, sich mitzuteilen, und Jakob tut alles, um «ihn zu verleiten, sich ein wenig mir gegenüber auszusprechen ». ${ }^{18}$ Und Jakobs Rechnung scheint aufzugehen; er scheint das Ziel seiner Strategie zu erreichen: ein Freundschaftsband $\mathrm{zu}$ knüpfen. Es ist mehr noch das Ziel Benjamentas selbst, der seinen Eleven an sich binden will ${ }^{19}$; das Ende des Romans bestätigt auf den ersten Blick die Bildung eines Freundes- oder Brüderpaars. Während Joseph sich von Tobler trennt, werden diese beiden zusammen die Abenteuer bestehen, die ihrer harren: «' [...] erlauben Sie mir, Ihnen zu sagen, daß ich mich entschlossen habe, mit ihnen zu gehen, wohin Sie wollen.' Wir gaben einander die Hand, und das bedeutete viel. » (JvG 164)

Wohnt man infolgedessen am Ende des Romans einer «Verwandlung von Herrschaft in Brüderlichkeit ${ }^{20}$ bei, wie Peter von Matt dies postuliert? Von Matt unterscheidet deutlich zwischen den Beziehungen der Protagonisten mit der Gesellschaft in ihrer Gesamtheit, die zum Scheitern verdammt sind, und den Beziehungen der Protagonisten untereinander, die gelingen, aber von der Umgebung abgetrennt sind: «[...] die privat-intime Sozialisation [sabotiert] ihre Ausweitung ins Öffentliche. ${ }^{21}$ Doch selbst die privat-intime Sozialisation ist sie wirklich gelungen? Ist es nur ihre Ausweitung ins Öffentliche, die nicht realisiert wird? Den Beweis, dass sich zwischen den beiden Menschen eine wahre Brüderlichkeit etabliert, bleibt der Text meines Erachtens schuldig. Andreas Solbach bemerkt zu Recht: «[...] in dem $\mathrm{Maße}$, in dem der Herr sich dem Knecht nähert, entzieht sich dieser. ${ }^{22}$ Konkret zeigt sich

\footnotetext{
${ }^{16}$ Daraus erklärt sich das häufig anzutreffende Wortfeld der Familienbeziehungen. Vgl. zum Beispiel JvG 107: «[...] manchmal ist mir, als seiest du mein junger Bruder [...]. » In diese Richtung entwickelt sich übrigens auch das Verhältnis zu Fräulein Benjamenta, der Schwester des Direktors. Diese redet Joseph etwa wie folgt an: «Laß mich dich, junges Menschenherz, ganz ins geschwisterliche, ins lächelnde Vertrauen ziehen. » (JvG 145)

${ }^{17}$ Vgl. zum Beispiel JvG 129: « Doch ich liebe zum ersten Mal einen Menschen. »

${ }^{18}$ JvG 45: « [...] der Schmerz, den mir der Unwille dieses seltsamen Mannes verursacht, ist nur klein gegen die bebende Begierde, ihn zu verleiten, sich ein wenig mir gegenüber auszusprechen. »

${ }^{19} \mathrm{Vgl}$. JvG 128: « [...] bleib du lieber noch. »

${ }^{20}$ Peter von Matt : «Die Schwäche des Vaters und das Vergnügen des Sohnes » (Anm. 3), S. 197.

${ }^{21}$ Ibid.: «Die Reise geht [...] ins gesellschaftliche Nichts. Damit sabotiert die privat-intime Sozialisation ihre Ausweitung ins Öffentliche. »

22 Andreas Solbach : «Herr und Knecht bei Kafka, Walser und Hegel », in : Zeitschrift für deutsche Philologie 106 (1987), S. 233. Äußerst zweifelhaft erscheint jedoch Solbachs Versuch, diese Reaktion dadurch zu erklären, dass Jakob in einer Strategie der Selbstauslöschung sein Ich nicht dem Mitmenschen gegenüber öffnen, sondern
} 
das in der Kälte, die Jakob gerade in jenen Augenblicken zeigt, da Benjamenta ihm seine Freundschaft anbietet. Auf die insistierenden Fragen des Direktors: «Willst du ganz, ganz bei mir bleiben? [...] wollen wir beide, du der Kleine, ich der Große, zusammen versuchen, wie wir das Leben bestehen? »( JvG 149) antwortet Jakob zwar bejahend, aber mit so wenig Enthusiasmus, dass das Ja nicht wenig entwertet wird: "Meiner Ansicht nach hat die Beantwortung dieser Frage noch Zeit, Herr Vorsteher. Aber was Sie sagen, interessiert mich, und ich werde mir die Sache, etwa bis morgen, überlegen. Doch glaube ich, daß ich mit ja antworten werde. » (Ibid.)

Es hat den Anschein, dass Jakob sich durch den Ausbruch von Freude, das Offenlegen der Liebe durch den anderen bedroht fühlt, und das Kühle in seinen Worten gewinnt eine Färbung der Angst in diesem Passus: «Es überlief mich kalt angesichts der überströmenden Zärtlichkeit und Lebenslust, die aus den Augen des Mannes hervorbrachen. » (JvG 157) Diese Gefühlskälte verleitet ihn manchmal zu einem Verhalten, das Benjamenta erniedrigen würde, wenn er es sähe: Durch die geschlossene Tür streckt ihm Jakob lachend die Zunge heraus und das, nachdem er soeben vom anderen ins Vertrauen gezogen wurde und er dessen Liebeerklärung angehört hatte. ${ }^{23}$ Man könnte sagen: gerade deswegen. Denn es geht ihm wohl hauptsächlich darum, sich gegen die Herzensergießungen Benjamentas zu schützen, die eine Autonomie gefährden, an welcher Jakob über allen anderen Dingen gelegen ist. ${ }^{24}$ Das kommt in aller Deutlichkeit zum Ausdruck, als er über seine Beziehung zu Fräulein Benjamenta spricht (aber was er sagt, betrifft ganz allgemein seine Art, zwischenmenschliche Beziehungen zu beurteilen): «Wir gehörten zusammen. Natürlich mit Unterschied. Doch wir stunden uns mit einmal sehr nahe. Immer, immer aber mit Unterschied. Ich hasse es geradezu, so gar wenig oder gar keine Unterschiede zu empfinden.» (JvG 98) Deshalb führt jede Annäherung bei ihm sogleich zu einem Schritt zurück und Freundschaft wird zu einer « verbotenen Frucht». Wenn er von sich und Benjamenta sagt, sie « scheuen vor der offenen Sprache zurück » (JvG 105), so spricht er wohl in erster Linie von sich selbst: Er selbst kann sich nicht zu dem Entschluss durchringen, den Benjamenta in seinem Glück, einen Partner gefunden zu haben, zu fassen wagt. So wird verständlich, warum seine Antwort so vage, so ausweichend ist, wenn es darum geht, die Beziehung zwischen dem Direktor und dem Eleven in Begriffe zu fassen: «Sind wir Freunde? Sind Herr Benjamenta und ich Freunde? Jedenfalls besteht zwischen uns beiden ein Verhältnis, aber was für eins? Ich verbiete mir, mir das erklären zu wollen.» (JvG 95) Trotz seiner Sehnsucht nach Kontakt, Zusammenhalt, menschlicher Wärme ist er unfähig, sich in eine ehrliche Beziehung einzubringen. ${ }^{25}$

es jeder individuell-persönlichen Reaktionsmöglichkeit berauben wolle. Vgl. Ibid.: «Jakob identifiziert sich mehr und mehr mit der bewußtlosen Automatik des Dienens, wie er sie bei seinem Mitschüler Kraus findet. »

${ }^{23}$ JvG 130: «Wieder horchte ich draußen im Korridor am Schlüsselloch, und wieder blieb es ganz still. Ich streckte sogar ganz läppisch und echt zöglinghaft die Zunge heraus, und dann mußte ich lachen. »

${ }^{24}$ Auf den kindlichen, ja kindischen Aspekt dieses Verhaltens, der für Walser und viele seiner Protagonisten so typisch ist, kann hier nicht eingegangen werden (vgl. unter anderem Gerd Hammer : Robert Walser: Momente des Kindlichen, Frankfurt a. M. : Afra-Verlag, 1989).

${ }^{25} \mathrm{Vgl}$. die ironische Reduktion der Freundschaft auf ein Vergnügen, eine Zerstreuung, der man, so lässt sich folgern, nach Belieben ein Ende setzen kann: «Mit einem edlen Menschen Freundschaft schließen und Turnen, das sind wohl zwei der schönsten Sachen, die es auf der Welt gibt. Tanzen, und einen Menschen finden, der mir Achtung entlockt, ist mir ein und dasselbe. » (JvG 119) 
Letztendlich scheint es Jakob unmöglich zu sein, sich einem Räsonnement in Begriffen von Herrschaft und Unterlegenheit zu entziehen - genauso wie im Gehülfen wohnt der Leser Machtkämpfen innerhalb des Lagers der Schwachen bei. In diesem Sinne können auch Jakobs Provokationen verstanden werden. Gewiss, sie dienen dazu, Benjamenta aus der Defensive zu locken: «In mir lebt eine sonderbare Energie, das Leben von Grund auf kennen zu lernen, und eine unbezwingliche Lust, Menschen und Dinge zu stacheln, daß sie sich mir offenbaren. Hier fällt mir Herr Benjamenta ein. ${ }^{26}$

Provokation als Strategie zur Überwindung menschlicher Barrieren: diese Interpretation steht im Widerspruch zu der Tatsache, dass Jakobs Sticheleien auch über den Moment hinaus andauern, wo Benjamenta sich entschließt, ihm sein Herz zu öffnen. ${ }^{27}$ Jakob spielt den aktiven Part in dieser Beziehung und erscheint insofern als der Dominierende gegenüber dem Direktor, der 'mit sich geschehen lässt', der den Einfluss des anderen fast willenlos - wenn auch mit einem punktuellen Aufbäumen seines Zorns und seiner physischen Gewalt - über sich ergehen lässt. Jakob, der sich seiner Macht bewusst wird, jubiliert und gibt sich Träumen von Allmacht hin, wie in der Szene, in der er Benjamenta die Zunge herausstreckt: «Wenn ich so lache, nun, dann steht nichts mehr über mir. Dann bin ich etwas an Umfassen und Beherrschen nicht zu Überbietendes. Ich bin in solchen Momenten einfach groß. » (JvG 130)

Benjamentas Größe dagegen ist nicht jene, die Jakob bei seinem Eintritt ins Institut zu gewahren glaubte - und die er ihm während des ganzen Romans bis in die letzten Seiten hinein zuspricht. Es ist wohl unmöglich zu bestimmen, welchen Anteil Jakobs mangelnde Klarsicht an der Deformation des wahren Kräfteverhältnisses zwischen Lehrer und Eleven hat (dies nicht zuletzt deswegen, weil Jakob von Gunten keine 'realistische' psychologische Studie ist); auf jeden Fall ist in diesem Text eine Dekonstruktion von Benjamentas Macht in Gang, die sich der zweideutigen Gefühle Jakobs bedient. Jedes Mal, wenn Benjamentas Allgewalt gepriesen wird, relativiert der Text in der Folge diese Aussage - und kehrt sie nicht selten um:

Und dann ist er ja so allmächtig, und ich Zögling, wie winzig bin ich. Still, nicht von Allmacht reden. Man irrt sich stets, wenn man große Worte in den Mund nimmt. Herr Benjamenta ist der Erschütterung und Schwäche so fähig, so sehr fähig, daß es beinahe zum Lachen, vielleicht sogar zum Grinsen ist. (JvG 140)

\footnotetext{
${ }^{26}$ JvG 114. Derselbe Gedanke taucht im Grunde schon in dieser Passage (JvG 45) auf: « Ja, und deshalb, um etwas herauszukriegen aus all diesem Geheimnisvollen, reize ich ihn, damit ihm etwas wie eine unvorsichtige Bemerkung entfahre. » Es ist diese Strategie, die auch die Vorwürfe bezüglich der im Institut angewandten Pädagogie motiviert, mit denen Jakob den Direktor provoziert: «Man lernt hier gar nichts [...]. » (JvG 19) Darauf weist schon Michael Pleister («Jakob von Gunten: Utopie oder Resignation?», in : Sprachkunst 23 (1992), S. 100) hin: «In Wirklichkeit scheint Jakob den Disput mit Benjamenta nur herbeigeführt zu haben, um den Schulleiter näher kennenzulernen [...]. »

27 So ist die eben zitierte Passage aus Seite 114 - der Tagebuchlogik entsprechend - zeitlich später niedergeschrieben als der Bericht von Benjamentas Geständnis: « Ich bin ein abgesetzter König. » (JvG 107)
} 
Und auch der Ausblick in die Zukunft erlaubt es nicht, die fragil oder inexistent gewordene Machtposition zu konsolidieren. Gewiss, Benjamenta erklärt Jakob gegenüber selbstsicher: « Und nun bin ich wieder, das heißt fange an, wieder ich selber zu sein, und es ist mir, als hätte ich eine Million geerbt, ach was, Million geerbt, nein, es ist mir, als wäre ich - - zum Herrscher erhoben und gekrönt worden. » (JvG 159) Allerdings korrigiert bzw. relativiert er diese Aussage im folgenden Satz, wo er seinem Eleven anvertraut, er habe ein "gleichsam verbrannte[s] und verkohlte[s] Gemüt ${ }^{28}$. Selbst am Ende des Romans wird das «verkohlte Gemüt» nicht als Charakteristik der persönlichen Vergangenheit erwähnt - das Gemüt scheint immer noch verkohlt zu sein oder, um ein anderes Bild zu benutzen, die Verletzungen, die das Leben ihm zugefügt hat, schmerzen immer noch und Jakob neigt eher dazu, Salz in die Wunden, die er heilen soll, zu streuen.

Die Vorstellung einer Verwundbarkeit der Großen wird übrigens auch durch biblische Namen suggeriert, die auf den ersten Blick das Riesenhafte an Benjamenta unterstreichen sollen. Indem er seinen Direktor als «Samson », als « Riesen Goliath » beschreibt ${ }^{29}$, wählt Jakob - und wir befinden uns wohlgemerkt gegen Ende des Romans - zwei Männer von beeindruckender physischer Kraft, die durch schwächere, aber klügere Menschen bezwungen werden (nämlich Delila bzw. David). Jakobs Provokationen sind wie Schüsse aus der Steinschleuder, gegen die Benjamenta machtlos ist.

Man sollte folglich auch nicht die Vitalität überschätzen, die Benjamenta manchmal in sich vergraben fühlt. ${ }^{30}$ Anscheinend handelt es sich um (selbst-)zerstörerische Triebe, die ohne Jakobs befreiende Hilfe nicht in positive Kräfte verwandelbar sind. Jakob ist es, der am Ende des Romans wie die personifizierte Vitalität erscheint; er ist es, der die Devise verkörpert: « Ich fühlte, daß das Leben Wallungen verlangt, nicht Überlegungen. » (JvG 164) Benjamenta ist keineswegs « eine schöne, volle Natur» (DG 247), wie Frau Tobler dies von ihrem Mann sagt, scheint aber zu ahnen, dass Jakob es auf seine Art ist - trotz der Erziehungsmethode des Instituts, wo es darum geht, « die Natur zu unterdrücken » (JvG 103); ja, dieser Unterricht hat ganz im Gegenteil die (heimliche) Vitalität dieses Schülers gestärkt. So könnte man zumindest die Worte interpretieren, die Benjamenta an ihn richtet: «[...] sei so, wie du bist und hier wurdest [...]. » (JvG 128) Und diese Vitalität ist es, die Jakob Benjamenta angeblich einflößt: «Mit dir, du Gemüt von einem Jungen, ist frisches, ist überhaupt erst Leben über mich und in mich hineingekommen. » (JvG 156) Selbst wenn man darin theoretisch eine Instrumentalisierung Jakobs und damit das Indiz einer Überlegenheit Benjamentas sehen könnte ${ }^{31}$, sind doch die Elemente des Texts, die die Position des Direktors

\footnotetext{
${ }^{28} \mathrm{JvG} 159$ f.: «Allerdings kommen mir immer wieder die dunklen, grauenhaft dunklen Stunden, wo mir alles schwarz vor den Augen und hassenswert vor dem gleichsam, versteh mich, verbrannten und verkohlten Gemüt wird, und in solchen Stunden zwingt es mich, zu zerreißen, zu töten. »

${ }^{29}$ JvG 142: «Er glich einem Simson [...]. [...] ich glaube, ich habe ihn, den großen Herrn Benjamenta, den Riesen Goliath, sogar in den Finger gebissen. »

${ }^{30}$ Vgl. Jochen Greven : « Nachwort », in JvG 177: «Den vitalen Kräften andererseits, diesem Elementaren, das den Vorsteher mit gefesselter Gewalt erfüllt wie einen Vulkan und das Jakob in ihm wieder befreit, hat Walser nie sonst in seinem Werk eine so positive, vertrauende Gestaltung gegeben wie hier. »

${ }^{31}$ Jakob fragt sich: «Will er sich jetzt etwa an mir ausleben? » (JvG 147) Am entschiedensten vertritt KlausPeter Philippi eine solche Deutung, nach der Benjamenta als Sieger aus diesem Machtkampf hervorgeht. Vgl. «Robert Walsers Jakob von Gunten" », in: Provokation und Idylle. Zum Werk Robert Walsers [= Der Deutschunterricht 23 (1971), Beiheft 1], S. 64 ff.
} 
(diskret) herabwerten (indem sie ihn als von Jakob abhängig zeigen) viel zahlreicher. ${ }^{32} \mathrm{Ja}$, es ist Benjamenta selbst, der einräumt, dass er von Jakob «fast, fast abhängig » (JvG 129) ist und mit der Relativierung durch das « fast, fast » versucht, sich selbst über das wahre Ausmaß seiner Unterlegenheit hinwegzutäuschen.

Und doch scheint diese Unterlegenheit sogar in den Namen des Direktors eingeschrieben zu sein: Mit Bezug auf die Bibel beschreibt Jakob ihn zu Beginn des Romans als Patriarchen, aber je deutlicher die Schwäche Benjamentas zutage tritt, desto eindringlicher stellt sich für den Leser die Frage, ob der Name Benjamenta nicht eher die Kleinheit des jüngsten ${ }^{33}$ der Brüder evoziert, von denen im Alten Testament die Rede ist. Und wenn man bedenkt, dass diese Brüder von einem Vater namens Jakob abstammen, liegt doch die Interpretation nahe, es handle sich hier um ein Augenzwinkern, mit dem das bisher Gesagte bestätigt wird: Auch in Walsers Roman ist Jakob derjenige, der Benjamin / Benjamenta das Leben spendet - und der es ihm nähme, wenn er ihn verließe, wie Letzterer gesteht: «Höre, mich würde dein Weggehen schmerzen, es würde mir eine Wunde, eine ganz unheilbare, beibringen, es würde mich fast töten. ${ }^{34}$

So wie man sich fragen kann, wer in diesem Paar der Vater ist und wer der Sohn, so kann man auch nach der tieferen Bedeutung des den Roman beschließenden Bildes fragen: nämlich der Vision vom Ritter und seinem Knappen, die in die Welt hinausziehen ${ }^{35}$ - wieder ein Symbol der Beziehung, die zwischen Benjamenta und Jakob herrscht. Natürlich sind die beiden als entfernte Verwandte Don Quichotes und Sancho Panzas aufzufassen (Walser bewunderte Cervantes' Roman $^{36}$ ), die ihren Hirngespinsten nachjagen und ihrer eigenen Gesellschaft entfremdet sind. Sancho Panza ist es, der seinem Herrn in vielen Dingen überlegen ist, ihm in allen schwierigen Lebenslagen zur Seite steht und das Minimum an Kontakt mit der Realität zustande bringt, das zum Überleben des Paars notwendig ist. Folglich ist dieser Bezug auf Cervantes' Meisterwerk ein weiteres Indiz für die Existenz eines Ungleichgewichts zwischen den beiden Gestalten, eines Ungleichgewichts, das mit jedem Gedanken an zwischenmenschliche Harmonie unvereinbar ist und jeden Versuch einer dialektischen Interpretation unterminiert, der zufolge eine «Aufhebung » der Gegensätze zustande käme (siehe z.B. Christian Angerers Sicht, eine «freundschaftliche Beziehung » sei «mit einer hierarchischen Struktur verknüpft »). ${ }^{37}$ Es darf auch bezweifelt werden, ob die

\footnotetext{
${ }^{32}$ In diesem Sinn äußern sich einige Interpreten, darunter Mark Harman, der das Ende des Romans folgendermaßen kommentiert: «It is Benjamenta who now seems dependent on his former pupil [...]. » (Mark Harman, «Stream of consciousness and the boundaries of self-conscious fiction: the works of Robert Walser », in : Comparative Criticism 6 (1984), S. 125.)

${ }^{33}$ Dass Benjamenta den Eleven seinen «jungen Bruder » nennt, verrät insofern seine mangelnde Luzidität. Vgl. die schon zitierte Passage: «[...] manchmal ist mir als seiest du mein junger Bruder [...]. » (JvG 107)

${ }^{34} \mathrm{JvG} 129$. Benjamenta flicht zwar ein « fast » ein, doch auch diese Nuancierung (wie das kurz vorher erwähnte « fast, fast ») lässt sich als verzweifelter und unbeholfener Versuch deuten, das zu verbergen, was zu gestehen er nicht umhin kann. Jakob von Gunten ist ein Roman, in dem offene Liebe unmöglich und doch auch lebensnotwendig ist, wie aus den Worten von Benjamentas Schwester zu folgern ist: «Jakob, ich sterbe, weil ich keine Liebe gefunden habe. » (JvG 145)

${ }^{35}$ Vgl. JvG 163: « Ich war immer der Knappe, und der Vorsteher war der Ritter. »

${ }^{36}$ Für Walser ist Cervantes “ Don Quichote «der beste und größte Roman der Weltliteratur » (Robert Walser : Briefe, hrsg. von Jörg Schäfer, Frankfurt a. M. : Suhrkamp, 1979, S. 134).

37 Christian Angerer: Rollenspiele. Soziales Rollenverhalten und Identitätsverweigerung in Robert Walsers Texten der frühen und der Berliner Zeit, Stuttgart : Heinz, 1995, S. 151.
} 
Rolle des Knappen für Jakob eine Absage an die persönliche Freiheit bedeutet, wie in der Kritik gesagt wurde. ${ }^{38}$ Die Unterordnung dürfte auch hier eine ironische, gespielte sein, so wie sie es auch beim Schüler Jakob (wie schon beim Schüler Fritz Kocher) trotz gegenteiliger Behauptungen immer ist. Und diese Unterordnung ist hier aufmüpfiger denn je. Schlägt der Herr einen schärferen Ton an, so geht dieser Schuss nach hinten los, weil er das Weiterbestehen von Uneinigkeit und seine eigene geistige Abhängigkeit von Jakob unter Beweis stellt, wie in folgendem Abschnitt: «[...] kläre mich sofort auf. Sofort, hast du verstanden! » (JvG 156 f.)

Der Roman beschreibt also einen unausgesetzten Kampf, in dem Benjamenta, seine Niederlage ahnend, sich verzweifelt zu wehren sucht - gefangen, wie er ist, zwischen dem Wunsch, sich anzuvertrauen, und dem Streben, seine eigene hypothetische Macht zu sichern. Jakobs jubilierende Worte «Von diesem Augenblick an war etwas Bindendes zwischen uns getreten » und «Herr Benjamenta achtet mich» (JvG 94) dürfen dabei nicht zu der Schlussfolgerung verleiten, es gebe eine «innere Entwicklung», die zu «gegenseitige[m] Anerkennen » führe, wie Andreas Solbach behauptet. ${ }^{39}$ Wie prekär eine solche Folgerung ist, zeigt ein Blick auf die Fortsetzung des Passus, auf den sich Solbach stützt; es mag zwar eine «innere Entwicklung » bei Benjamenta geben (insofern er Jakobs Freundschaft gewinnen will), damit setzt aber noch nicht der Beginn einer wahren Freundschaft ein, denn Jakob weigert sich, sich dem anderen zu eröffnen - aus Furcht, in einem Austausch, der immer noch als Kampf aufgefasst wird, den Kürzeren zu ziehen:

»Herr Benjamenta achtet mich«, sagte ich mir, und infolge dieser wie ein Blitz auf mich niederstrahlenden Erkenntnis fand ich es für schicklich, ja sogar für geboten, zu schweigen. Wehe mir, wenn ich ein einziges Wort gesagt hätte. Ein einziges Wort würde mich zum unbedeutsamen kleinen Eleven erniedrigt haben [...]. (JvG 94)

Ja, der Roman enthält zahlreiche Passagen, in denen die Gefahr einer Öffnung gegenüber dem anderen heraufbeschworen wird, sei es nun Jakob, der seine Versuche, Benjamenta zum Reden zu bringen, als List, wenn nicht als Falle, die er dem anderen stellt, beschreibt ${ }^{40}$, oder Benjamenta selbst, der bald die Gefahr, der er sich aussetzt, als verlockend bezeichnet ${ }^{41}$, bald Jakob anfleht, ihrem Dialog (durch Frechheiten) ein Ende zu setzen, weil er meint, in seinen Geständnissen zu weit gegangen $\mathrm{zu}$ sein. ${ }^{42}$ Dies soll nicht heißen, dass Jakob keine Freundschaft für Benjamenta empfände, aber dieses Gefühl darf nicht ausgesprochen werden. In einem auf Machtgewinn und -verlust ausgerichteten Verhältnis gälte dies als Zeichen der

\footnotetext{
${ }^{38}$ Vgl. Michael Pleister : «Jakob von Gunten: Utopie oder Resignation? » (Anm. 25): « Durch das Bündnis mit Benjamenta verliert Jakob zwangsläufig seine ursprüngliche Freiheit. »

${ }^{39}$ Andreas Solbach : « Herr und Knecht bei Kafka, Walser und Hegel » (Anm. 21), S. 233.

40 Jakob spricht von seiner «bebende[n] Begierde, ihn zu verleiten, sich ein wenig mir gegenüber auszusprechen. »(JvG 45)

${ }^{41} \mathrm{Vgl}$. JvG 107: «Es ist prickelnd reizvoll, sich dir gegenüber ein wenig schwach und weicher, als gewöhnlich, zu benehmen. »

${ }^{42}$ Vgl. JvG 94: « Jetzt, bitte, werde frech. »
} 
Schwäche ${ }^{43}$, das der andere ausnutzen könnte und das deswegen verborgen werden muss: «Ich heuchelte irgendwelche Kälte, irgendwelche Oberflächlichkeit, während ich doch am liebsten hätte in sein Gesicht lachen mögen, vor Freude. » (JvG 95) So gelingt es Jakob, Benjamenta in «Ketten zu fesseln ${ }^{44}$, wobei er in diesem Kampf explizit militärischen Wortschatz benutzt: « Es ist ein Vorpostengefecht gewonnen. » (JvG 53)

Gewiss, Jakob von Gunten schließt mit einem positiven, versöhnlichen Bild, einem Bild der Harmonie, das aber kaum glaubwürdig scheint. Michael Pleister kritisiert den von Naguib benutzten Begriff des «Anti-Bildungsromans » ${ }^{45}$ mit dem Argument, es vollziehe sich hier durchaus eine Bildung, aber: «Die konventionelle bürgerliche Bildung wird hier also negiert und durch eine andere ersetzt. ${ }^{46}$ Es handle sich also um einen « umgekehrten Bildungsroman ${ }^{47}$. Dem kann entgegnet werden, dass diese « umgekehrte Bildung », die auf Unterwerfung und Machtverzicht aus wäre, ebenfalls scheitert. Das Ende, auch wenn es auf den ersten Blick «euphorisch ${ }^{48}$ erscheint, ist letztlich genauso zweideutig, wie es das der anderen Walserschen Romane ist. ${ }^{49}$ Und es ist keineswegs erstaunlich, dass Jakob nicht sieht, oder nicht sehen will - das heißt verdrängt -, was wirklich zwischen ihm und Benjamenta abläuft. Es mangelt ihm an Hellsicht während des ganzen Romans - und er selbst thematisiert diesen Mangel in manchen Tagebucheinträgen. So leuchtet es nicht ein, dass es just am Ende anders sein soll, jetzt, wo er jeder Reflexion, jedem Versuch, sich Rechnung zu geben, abschwört: «Ah bah, laß das Deuten! »(JvG 162) Da «Gott [...] mit den Gedankenlosen [geht] » (JvG 164), kann sich Jakob in dem Glauben wiegen, er und Benjamenta seien «beide in Eines verschmolzen » (JvG 141). Aber der gesamte Roman veranlasst den Leser vielmehr, die Möglichkeit einer Rückkehr zur Naivität eines « unreflektierten, rein physischen Daseins » und der Wiedererlangung einer durch die « Rationalisierung der Lebenswirklichkeit verdorbenen » Authentizität ${ }^{50}$ in Zweifel zu ziehen. Die «positive Utopie einer totalen

\footnotetext{
${ }^{43}$ Siehe Benjamentas Worte: « Nicht wahr, junger Mensch, jetzt, nachdem ich mir vor dir eine Blöße gegeben habe, wirst du's wagen, mich mit Wegwerfung zu behandeln? » (JvG 94) Bezeichnend ist, dass der Begriff der 'Blöße', so wie er hier gebraucht wird, aus dem Wortschatz der Fechter stammt und folglich die Existenz einer kämpferischen Auseinandersetzung präsupponiert.

${ }^{44}$ Benjamenta nennt Jakob einen «[...] Schelm, der du mich mit so eigenartigen fröhlichen Ketten fesselst [...]. » (JvG 149)

${ }^{45}$ Nagi Naguib : Robert Walser. Entwurf einer Bewußtseinsstruktur, München : Fink, 1970, S. 84.

${ }^{46}$ Michael Pleister : «Jakob von Gunten: Utopie oder Resignation? (Anm. 25), S. 98.

${ }^{47}$ Ibid. S. 99: «Da sein Ziel in der Servilität und nicht in der Entfaltung seiner geistigen Anlagen mit Hilfe bürgerlicher Kulturgüter besteht, kann Jakob von Gunten schlicht als umgekehrter Bildungsroman bezeichnet werden, als Entwicklungsroman, dem die klassischen Merkmale fehlen. »

${ }^{48}$ Vgl. Dagmar Grenz : Die Romane Robert Walsers (Anm. 4), S. 205. Zu den Kritikern, die Jakobs Euphorie beim Wort nehmen, zählt neben Peter von Matt auch Tamara S. Evans (Robert Walsers Moderne, Bern / Stuttgart : Francke, 1989, S. 60).

${ }^{49}$ George Avery (Inquiry and Testament. A Study of the Novels and Short Prose of Robert Walser, Philadelphia : University of Pennsylvania Press, 1968, S. 69) spricht von einem «ambiguous, open end, typical to all three novels ». Dies gilt natürlich noch mehr für den Räuber-Roman. Vgl. Martin Jürgens, « Nachwort », in : Robert Walser : Der Räuber, Frankfurt a. M. : Suhrkamp, 1986, S. 200-207, und Karl Wagner : "Geld und Beziehungen. Walser - Musil - Rathenau », in : Klaus-Michael Hinz / Thomas Horst (Hrsg.), Robert Walser, Frankfurt a. M. : Suhrkamp, 1991, S. 329. - Eine kurze Präsentation der «kontroverse Deutungen des Romanendes » von Jakob von Gunten findet sich in Peter Utz : «Robert Walsers Jakob von Gunten. Eine 'Null'Stelle der deutschen Literatur», in: Deutsche Vierteljahrsschrift für Literaturwissenschaft und Geistesgeschichte 74 (2000),3, S. 507.

${ }^{50}$ Siehe Jochen Grevens (Existenz, Welt und reines Sein im Werk Robert Walsers. Versuch zur Bestimmung von Grundstrukturen, Diss., Köln 1960, S. 80) Erläuterung des '[G]unten' in Jakobs Familiennamen: «[...] die
} 
Freiheit ${ }^{51}$ kann nur existieren, weil Jakob willentlich die Augen vor den weiterbestehenden Machtstrukturen und vor allem -techniken verschließt - Strukturen, von denen er profitiert, Techniken, die er anwendet - ist er doch der heimliche Machthaber - und unter denen er zugleich leidet, da er als Herrscher den Status des Freundes (den er hat oder zumindest haben könnte) kompromittiert.

Man kann folglich nur Klaus-Peter Philippi beipflichten, wenn er feststellt, bei dem in die Wüste ziehenden Paar handle es sich um eine « irreale phantastische Einheit [...], in der sich, nun unreflektiert, die Macht- und Abhängigkeitsverhältnisse des Instituts fortsetzen" ${ }^{52}$, wobei allerdings präzisiert werden muss, dass die «Abhängigkeitsverhältnisse » nicht, wie Philippi meint, die sind, die uns der Anfang des Romans vorgaukelt. Nichtsdestotrotz kann von einer Kontinuität hierarchischer Strukturen gesprochen werden - was es uns erlaubt, Pleisters Konstatierungen zum Ende des Romans noch etwas weiter zu treiben: Dieser begnügt sich damit, das Fehlen von «Aussagen zu den sozialen Bedingungen » in einem Text, der « reine Vision » sei $^{53}$, festzustellen. Wenn die sozialen Bedingungen einer Existenz « in der Wüste » oder in Indien im Roman nicht präzisiert werden, so deswegen, weil diese am Ende heraufbeschworene Existenz 'nicht von dieser Welt' ist. Es handelt sich um eine abschließende Apotheose der antibürgerlichen Welt schlechthin, der Welt der Poesie, wie sie den antibürgerlichen Gestalten Jakob und Benjamenta zuzuordnen ist, während Pleister die Abreise der beiden in einem wörtlichen, zu wörtlichen Sinn nimmt, nämlich dem einer Reise in eine Weltgegend, wie sie tatsächlich in der äußeren Realität zu finden ist. Aber der Stoff, aus dem diese Welt gewoben ist, ist eben der Traum: «Will doch sehen, ob es sich in der Wildnis nicht auch leben, atmen, sein, aufrichtig Gutes wollen und tun und nachts schlafen und träumen läßt. » ${ }^{54}$ Der Traum, diese von Grund auf individuelle, asoziale Aktivität, vollzieht sich in einem Zustand sozialer Bewusstlosigkeit oder der Unkenntnis der gesellschaftlichen Basis, von der er sich nährt. Er erlaubt es Jakob, in seiner Blindheit gegenüber der wahren Beziehung zu Benjamenta fortzuleben. Jakob will seine Souveränität Benjamenta gegenüber nicht aufgeben und wird damit zu dem, der in dieser Beziehung die Zügel in der Hand hat - und er wird die so gewonnene Macht immer dann ausspielen, wenn es für ihn darum geht, die Freiheit, von Freundschaft und Liebe zu träumen, zu bewahren und auszukosten zu können - ohne auf die anderen, auf den anderen, Rücksicht nehmen zu müssen. Es ist diese seinem Existenzmodus inhärente Notwendigkeit, zwischenmenschliche Beziehungen stets in der Form hierarchischer Konkurrenz zu etablieren, die es dem

\footnotetext{
Rationalisierung der Lebenswirklichkeit verfälscht und 'verdirbt' dieses Leben. 'Unten' ist also auch das unreflektierte, rein physische Dasein [...]. »

${ }^{51}$ Martin Jürgens : Robert Walser. Die Krise der Darstellbarkeit. Untersuchungen zur Prosa, Kronberg im Taunus : Scriptor, 1973, S. 65.

52 Klaus-Peter Philippi : «Robert Walser. Jakob von Gunten» (Anm. 30), S. 69. Philippi zufolge nimmt Benjamenta die dominierende Position ein - auch am Ende noch.

${ }^{53}$ Michael Pleister : «Jakob von Gunten: Utopie oder Resignation?» (Anm. 25), S. 103: « Da keine Aussagen über die sozialen Bedingungen getroffen werden, unter denen sich Jakob und Benjamenta im neuen unmittelbaren Leben zurechtfinden, ist kaum anzunehmen, daß der Aufbruch etwas anderes als eine bloße Vision darstellt. »

${ }^{54}$ JvG 164. Vgl. dazu Peter Utz: «Robert Walsers Jakob von Gunten. Eine 'Null'-Stelle der deutschen Literatur » (Anm. 48), S. 507.
} 
Walserschen Protagonisten verbietet, solidarische und freundschaftliche Beziehungen einzugehen.

Als Fazit lässt sich deshalb festhalten, dass - um die eingangs benutzte Begrifflichkeit wiederaufzunehmen - tatsächlich von Mimesis im Vater-Sohn-Verhältnis gesprochen werden kann, diese Mimesis jedoch allenthalben ironisch - und damit allenthalben subversiv ist. Dabei ist das Modell in Walsers Texten, und besonders in Jakob von Gunten, zweistufig: So wie Tobler sich als geachteter bzw. zu achtender Bürger aufspielt, gibt auch Benjamenta - zu Beginn des Romans - sich den Anschein, er würde als Lieferant zukünftigen Dienstpersonals der bürgerlichen Gesellschaft zuarbeiten. Diese Vorstellung wird im Laufe der Romane subvertiert - doch ist die sich entpuppende Unbürgerlichkeit nicht der Zielpunkt von Walsers Schreiben, sondern die Folie einer zweiten, und zwar typisch Walser'schen, Subversion: Unterminiert wird die Vorstellung, Jakob könnte sich mimetisch dem Vorbild Benjamenta anschließen und sich mit ihm solidarisieren. Zwischen jenen, die sich aus der bürgerlichen Gesellschaft ausklinken wollen, entsteht nur in sehr begrenztem Maße Solidarität. Es gibt Machtkämpfe im Lager der Schwachen.

Damit gewinnt insbesondere auch Kommunikation eine zutiefst agonistische Dimension: Wenn Mimesis, die Nachahmung des Modells, sich als ironische Mimikry, die vorgespiegelte Solidarität sich als getarnte Rivalität entpuppt, darf auch der Sprachgebrauch selbst, und damit das Dialogische, als ironisch bezeichnet werden. Es geht auch hier - mehr als um das Zwiegespräch, den Austausch - um die Entblößung des anderen und die Verhüllung seiner selbst. In diesem Kontext ist Schweigen ein Machtinstrument. Benjamenta verwendet es zu Beginn (indem er vor dem sich vorstellenden Eleven ostentativ Zeitung liest), bevor Jakob den Spieß umdreht. Er antwortet nicht und wenn, dann mit betonter Langsamkeit und Zurückhaltung. « Ein einziges Wort würde mich [...] erniedrigt haben », heißt es an einer Stelle, wie wir gesehen haben.

Höchst bedeutsam sind dabei auch die Modi und Funktionen des Lachens, das nur ein einziges Mal als Zeichen zwischenmenschlicher Herzlichkeit zu verstehen ist - und in diesem Fall nur im Irrealis erscheint: « [...] am liebsten hätte ich in sein Gesicht lachen mögen vor Freude. » Tatsächlich ist es im Roman ein Zeichen der Selbstherrlichkeit Jakobs (angesichts von Benjamentas Schwäche), der Provokation sowie der Blockade: zur Verhinderung unwillentlicher Gefühlsmanifestationen - also zur Verhinderung eines echten Dialogs.

In gewissem Sinne - darauf soll abschließend noch hingewiesen werden - entspricht diese Strategie dem Programm Benjamenta'scher Erziehung: Es geht darum, die Natur zu unterdrücken. Gesteht der Institutsvorsteher, er habe eine «nicht zu beherrschende Vorliebe » für Jakob gefasst, so zeigt er auf seine Art, dass es um Herrschaft geht, und nicht zuletzt um die Herrschaft über die Gefühle, also um Affektkontrolle. Natur kann sich nur unterdrückt äußern - also indirekt. Und Indirektheit manifestiert sich gestisch, in erster Linie jedoch sprachlich. «Wir scheuen vor offener Sprache zurück », heißt es im Roman. Diese Scheu bewirkt, dass schon Gesagtes, Offengelegtes unentwegt zurückgenommen wird. Was hier im Zusammenhang der Vater-Sohn-Beziehung, und insbesondere der Beziehung zwischen Benjamenta und Jakob, herausgearbeitet wurde, ist konstitutiv für Walsers Schreiben im 
Allgemeinen und nicht zuletzt für die schriftstellerischen Techniken des Spätwerks, deren Eigentümlichkeit Christoph Bungartz in der Walserschen Formel des «zurückweichend Vorwärtsschreiten » komprimiert hat. ${ }^{55}$ Auch in der Beziehung zwischen Jakob und Benjamenta gibt es ein solches zurückweichend Vorwärtsschreiten, das mit dem Gedanken einer 'Urversöhnung' kaum zu vereinen ist.

${ }^{55}$ Christoph Bungartz : Zurückweichend vorwärtsschreiten. Die Ironie in Robert Walsers Berner Prosa, Bern e.a. : Peter Lang, 1988. 